\title{
1 The methylome and comparative transcriptome after high intensity sprint exercise in
}

\section{2 human skeletal muscle}

3

4 Mohd Firdaus Maasar 2, Daniel C. Turner 2,3, Piotr P. Gorski ${ }^{1,3}$, Robert A. Seaborne ${ }^{2,4}$, Juliette A. Strauss

5 5, Sam O. Shepherd ${ }^{5}$, Matt Cocks ${ }^{5}$, Nicolas J. Pillon ${ }^{6}$, Juleen R. Zierath ${ }^{6,7,8}$, Andrew T. Hulton ${ }^{9}$,

6 Barry Drust $10^{*}$, Adam P. Sharples $1,2,3^{*}$

$9{ }^{1}$ Institute for Physical Performance, Norwegian School of Sport Sciences (NiH), Oslo, Norway.

102 Stem Cells, Aging and Molecular Physiology Unit, Exercise Metabolism and Adaptation Research Group,

11 Research Institute for Sport and Exercise Sciences, Liverpool John Moores University, Liverpool, United

12 Kingdom.

$13{ }^{3}$ Institute for Science and Technology in Medicine (ISTM), School of Pharmacy \& Bioengineering, Keele

14 University, Staffordshire, United Kingdom.

$15{ }^{4}$ Centre for Genomics and Child Health, Blizard Institute, Barts and the London School of Medicine and

16 Dentistry, Queen Mary University of London, London, United Kingdom.

$17{ }^{5}$ Exercise Metabolism and Adaptation Research Group, Research Institute for Sport and Exercise Sciences,

18 Liverpool John Moores University, Liverpool, United Kingdom.

$19{ }^{6}$ Department of Physiology and Pharmacology, Karolinska Institutet, Stockholm, Sweden.

$207^{7}$ Department of Molecular Medicine and Surgery, Karolinska Institutet, Stockholm, Sweden

$21{ }^{8}$ Novo Nordisk Foundation Center for Basic Metabolic Research, University of Copenhagen, Copenhagen,

22 Denmark

$23{ }^{9}$ Department of Nutritional Sciences, School of Biosciences and Medicine, Faculty of Health and Medical

24 Sciences, University of Surrey, Guildford, UK.

$25{ }^{10}$ School of Sport, Exercise and Rehabilitation Sciences, College of Life and Environmental Sciences, University 26 of Birmingham, Birmingham, United Kingdom. 


\section{Abstract}

The methylome and transcriptome signature following exercise that is physiologically and metabolic relevant to sporting contexts such as team sports or health prescription scenarios (e.g. high intensity interval training/HIIT) has not been investigated. To explore this, we undertook two different sport/exercise relevant high-intensity sprint running protocols in humans using a repeated measures design of: 1) Change of direction (COD) versus; 2 ) straight line (ST) sprint exercise. We took skeletal muscle biopsies from the vastus lateralis 30 minutes and 24 hours post exercise followed by $850 \mathrm{~K}$ methylation arrays and comparative analysis with recent sprint and acute aerobic exercise metaanalysis transcriptomes. Despite matched intensity (speed $\mathrm{x}$ distance and number of accelerations/decelerations) between COD and ST exercise, COD exercise elicited greater movement (GPS Playerload ${ }^{\mathrm{TM}}$ ), physiological (HR), metabolic (lactate) as well as central and peripheral (differential RPE) loading compared with ST exercise. The exercise response alone across both conditions evoked extensive alterations in the methylome immediately post and $24 \mathrm{hrs}$ after exercise, particularly in MAPK, AMPK and axon guidance pathways. COD evoked a considerably greater hypomethylated signature across the genome compared with ST sprint exercise, particularly enriched in: Protein binding, MAPK, AMPK, insulin, and axon guidance pathways. A finding that was more prominent immediately post exercise. Comparative methylome analysis with sprint running transcriptomes identified considerable overlap, with $49 \%$ of the genes altered at the expression level also differentially methylated after COD exercise. After differential methylated region analysis, we discovered that VEGFA and its downstream nuclear transcription factor, NR4A1 had enriched hypomethylation within their promoter regions. VEGFA and NR4A1 were also significantly upregulated in the sprint transcriptome and meta-analysis of exercise transcriptomes. We confirmed increased mRNA expression of VEGFA, and considerably larger increases in the expression of canonical metabolic genes, PGC1- $\alpha$ and NR4A3, 3 hrs post COD vs. ST exercise. Overall, we demonstrate that increased physiological load via change of direction sprint exercise in human skeletal muscle evokes considerable epigenetic modifications that are associated with changes in expression of genes responsible for adaptation to exercise. These data imply that introducing changes in direction into high intensity running protocols could serve as an important modulator of a favourable epigenomic and transcriptomic landscape in response to exercise in athletes and trigger greater skeletal muscle remodelling through enhanced gene expression. 
Introduction

Epigenetic modifications, particularly DNA methylation, is associated closely with altering gene expression in response to exercise in human skeletal muscle 1. Genome-wide DNA methylation analysis after acute resistance exercise, training, detraining and retraining in humans has demonstrated that epigenetic modifications can be retained and therefore skeletal muscle possesses an epigenetic memory ${ }^{1}$. Resistance exercise preferentially reduces (hypomethylates) the DNA methylome 2, 3 and increases gene expression across the transcriptome 1 . With even greater hypomethylation observed if exercise training had been undertaken previously, such as following retraining 2, 3. Candidate genes demonstrating this epigenetic profile, such as UBR5 are important regulators of skeletal muscle mass ${ }^{4,5}$. Therefore, alterations in DNA methylation signatures are emerging as important drivers for the molecular response to exercise and subsequent physiological adaptation.

Methylation of DNA occurs most commonly in cytosine-guanine (C-G) base pairings called CpG sites. CPG islands are CpG rich locations that occur more frequently in gene regulatory regions, such as promoter or enhancer regions. In humans the majority of CpG sites are methylated (70-80\%) ${ }^{6,7}$. When methylation is present or increases (hypermethylation), particularly in promoters or enhancers, this prevents transcription factors from binding to these portions of the gene to enable gene expression ${ }^{8}$. In contrast, loss of methyl groups (hypomethylation) from these regions can enable/increase gene expression.

Aerobic exercise training alters the skeletal muscle methylome in humans ${ }^{9}$ and preferentially hypomethylates the genome in type-II diabetics ${ }^{10}$ and obese populations ${ }^{11}$. At the targeted gene level, acute aerobic exercise, particularly at higher intensities ( $80 \%$ vs. $40 \% \mathrm{VO}_{2}$ max) hypomethylates PGC-1 $\alpha$, a well-established regulator of mitochondrial biogenesis. With the same trend observed for associated metabolic pathway genes; TFAM, PDK4 and PPAR- $\delta{ }^{12}$, together with a corresponding

100 increase in their gene expression ${ }^{12}$. However, there is limited analysis of DNA methylation across the 101 genome after acute aerobic exercise in healthy human skeletal muscle. Furthermore, there is no temporal assessment of the methylome post-acute aerobic exercise, such as after $24 \mathrm{hrs}$ or after

103 alterations in exercise intensity. Finally, the methylome signature following exercise that is 104 physiologically and metabolic relevant to sporting contexts such as team sports or health prescription scenarios (e.g. high intensity interval training/HIIT) in the general public has not been investigated. 
In the present study, we devised a repeated measures sprint shuttle running protocol in humans that was physiologically relevant to exercise movements within many sporting contexts and health prescription settings. In the design there were two different sprint shuttle trials: 1) Change of direction (COD) and, 2) straight line (ST) sprint exercise that were both matched for classically defined intensity

110 measures (speed $\mathrm{x}$ distance) and the number of acceleration and deceleration movements. However, 111 despite this matched intensity between trials, in the COD exercise protocol greater movement (GPS 112 Playerload ${ }^{\mathrm{T}}$ ), physiological (HR), metabolic (lactate), subjective central and peripheral (differential 113 RPE) loading was elicited compared with ST sprint running. Therefore, the comparison of COD versus 114 ST exercise represented a suitable model to investigate the time-course of the DNA methylome 115 following increased loading exercise in human skeletal in a physiologically relevant model. Therefore, 116 we first aimed to investigate differential DNA methylation across the genome between these two trials 117 with dedicated genome-wide analyses for enriched gene ontologies, pathways and chromosomal 118 regions 30 minutes and $24 \mathrm{hrs}$ after COD versus ST exercise. Furthermore, we undertook comparative 119 analysis with recently published transcriptome data sets after acute sprint exercise in humans ${ }^{13}$, and 120 also with recent meta-analysis that combines transcriptome data from 66 published data sets in the most comprehensive exercise transcriptome profiling to date ${ }^{14}$. Subsequently, the secondary aim was to determine if the alterations across the methylome between COD versus ST exercise were also seen at the transcriptome level. With the ultimate aim of identifying epigenetic regulators in the response to acute exercise at the pathway and candidate gene level. Overall, the present study demonstrated that increased physiological load, incorporating changes of direction into sprint shuttle exercise in human skeletal muscle, evoked an extensive epigenetic signature that was associated hypomethylation and with changes of expression of gene pathways responsible for the adaptation to exercise (AMPK / MAPK / insulin/ axon guidance / protein binding pathways), as well as genes at the candidate level (VEGFA, NR4A1, NR4A3 and PGC1- $\alpha$ ). This study therefore suggests that introducing changes of direction into running protocols could serve as an important modulator in promoting a favourable epigenomic and transcriptomic response to acute exercise. Finally, an applied implication of the study suggests that by incorporating changes of direction into exercise regimes overtime (COD training) may ultimately improve performance within a sporting context or health outcomes of populations with dysfunction in the identified (MAPK, MAPK, insulin) gene pathways. 
Methods

Experimental overview

143 Following preliminary visits to the laboratory to complete screening and pre-test

144 assessments/familiarisation a repeated measures design was used for all participants to complete two

145 experimental trials; a 180-degree change of direction (COD) performed over a distance of $5 \mathrm{~m}$ to

146 volitional exhaustion and a repeated straight line $5 \mathrm{~m}$ sprint protocol (ST) performed at the same

147 intensity (running speed) until an equivalent distance to that covered in COD was completed (speed:

$1483.2 \pm 0.1 \mathrm{~m} . \mathrm{s}^{-1}$; distance: $594 \pm 35 \mathrm{~m}$ ). The individual responses to these exercise bouts were described

149 from a movement (GPS player load), physiological (HR), metabolic (lactate) and subjective perspective

150 (central and peripheral fatigue / rating of perceived exertion -RPE) to holistically characterize the 'load'

151 demand associated with the activity. Muscle biopsies were obtained from the lateral portion of the

152 vastus lateralis muscle for genome wide DNA methylation analysis, comparative transcriptome and

153 targeted gene expression, described in detailed below.

Participants

156 A group of five healthy well-trained, male team sports players (age: $26 \pm 2$ yrs; height: $1.7 \pm 0.1 \mathrm{~m}$; weight: $76.3 \pm 11.5 \mathrm{~kg}$ ) were recruited to participate in this study. After giving informed consent, all participants were screened using physical activity and readiness to exercise questionnaire and a prebiopsy medical assessment checklist. The information was used by the research team and a qualified medical practitioner to confirm inclusion criteria and eligibility for the current study. All participants included were free from any musculoskeletal injuries for the last six months. Participants were required to refrain from strenuous exercise 24 hours prior to testing, alcohol for 48 hours prior to exercise and caffeine for 12 hours prior to exercise. Ethical approval was granted from the institutional ethics review board/committee for the study (ref. 19/SPS/028).

167 Max Running Speed Test

168 All participants attended the laboratory, a maximum of two occasions, to complete a pre-test 169 assessment to determine the running speed to be used in the experimental protocols and to complete 170 familiarisation with the relevant measurement tools. Each participant was required to perform a $6 \mathrm{x}$ $1715 \mathrm{~m}$ shuttle run with a 180-degree change of direction at their self-chosen maximal speed. These runs 172 were performed until the speed at which the shuttles were completed demonstrated a coefficient of 173 variation of $5 \%$ or lower. This intensity was also re-evaluated on a second visit to ensure that 
174 participants could re-produce the required running speed identified in the first session to the same 175 accuracy $\left(3.21 \pm 0.1 \mathrm{~m}^{\mathrm{s}} \mathrm{s}^{1}\right)$. This speed was then used for the completion of all subsequent shuttle 176 runs, programmed into a computer derived audible beep. All participants also performed 10 177 repetitions in a preliminary trial of COD with 30-second rest in between repetitions. This acted as 178 familiarisation to both the movement patterns required for the protocol and the approach to be used 179 to evaluate the subjective responses to exercise.

180

\section{Experimental exercise protocols}

182 Participants performed two experimental protocols following an overnight fast; COD and ST on 183 separate occasions separated by a minimum of 2 weeks. All participants completed the COD protocol

184 first followed by ST. Both protocols were preceded by the completion of a standardised $10 \mathrm{~min}$ warm185 up which consisted of 5 min slow running to elevate HR. This activity was followed by dynamic and 186 static stretching to increase range of movement and readiness for the specific movement pattern of 187 the running protocol. The warmup was led by the researcher. The intensity (running speed) of COD 188 was controlled using a computer derived audible beep determined based on the maximum individual 189 speed collected during the pre-test assessment. After each repetition $(6 \times 5 \mathrm{~m}$ runs separated by a $190180^{\circ} \mathrm{COD}$ ), a 30 s rest period was completed. COD was performed until voluntary exhaustion (defined 191 as the point at which the participant could no longer complete the required shuttle distance at the 192 pre-determined speed). The ST sprints were performed at an equivalent intensity to that used for 193 COD. ST sprints required individuals to perform repeated $5 \mathrm{~m}$ sprints in a forward direction over a 30 $194 \mathrm{~m}$ course. To maintain a similar acceleration and deceleration profile to that of the COD participants 195 were required to come to a stop and then immediately set off again after each $5 \mathrm{~m}$ sprint. Computer 196 derived audible beeps were again used to "pace" the completion of the bouts of activity. Participants 197 maintained ST sprinting until the volume of activity matched that completed at voluntary exhaustion 198 in the COD trial.

200 Dependent variables associated with movement, physiological and subjective outcomes were 201 recorded to comprehensively describe the exercise in COD and ST and its associated demand. Prior to 202 the exercise, an athlete tracking unit that included a micro-electrical mechanical device and global 203 positioning system (Catapult S5, Australia) was fitted into a purpose made vest and placed on each 204 participant so it was positioned between the shoulder blades. All participants used the same tracking 205 unit throughout the testing session to avoid inter-unit variability of the measurement ${ }^{15}$. A short-range 206 radio telemetry device to monitor heart rate was also fitted (Polar T31 Kempele, Finland) according to 207 manufactures guidelines. Both movement and heart rate were recorded continuously throughout the 
exercise session. Following the completion of the experimental protocol, data was downloaded using manufacturers software (Catapult Openfield software pack) to obtain the peak heart rate observed in

210 COD and ST trials and the peak Playerload ${ }^{\mathrm{TM}}$ (the instantaneous rate of change of acceleration divided

211 by a scaling factor, used as an indicator of the overall movement demand ${ }^{15}$ ). During both experimental

212 protocols between the 30s rest periods between sprints, participants were asked to self-rate their

213 differential RPE for both local (legs) and central (breathlessness) exertion using a Centimax 100 scale

$214{ }^{16}$. This data provided a subjective evaluation of the central and peripheral demand associated with

215 the trials. The peak differential RPE for both legs and breathlessness were used to compare subjective

216 ratings between COD and ST. Capillary blood lactate was determined at rest (baseline) and post

217 exercise from a fingertip blood sample for each participant. Following a puncture made using a lancet

218 (Accu-Chek, Safe-T Pro Plus) a blood sample was drawn from the fingertip, using $20 \mu$ l blood capillary

219 tubes. The blood sample was immediately placed and mixed into the lactate haemolysing solution

220 cup, to prevent blood sample from clotting. All samples were analysed using Biosen C-Line "EKF

221 diagnostic" device. Pre-blood lactate sample was drawn at rest before the warm-up, and post-blood

222 lactate was drawn 5 minute following the completion of the exercise trial.

\section{Skeletal muscle biopsies}

225 Muscle biopsies were taken from the lateral portion of the vastus lateralis muscle under local 226 anaesthetic with Marcain using the conchotome technique. Due to ethical considerations of multiple 227 biopsies from the same leg over a short time period, within each trial muscle biopsies were taken from 228 both legs (detailed below). Upon arrival at the laboratory under overnight fasted conditions, the preexercise muscle biopsy was collected from the non-dominant leg. Post-exercise muscle biopsies were

230 taken (from the dominant leg) 30 minutes following the completion of COD and ST exercise. A final 231 biopsy was taken 24-hour post-exercise again following an overnight fast (from the non-dominant 232 leg). EMG analysis confirmed that there were no significant differences in muscle activation between 233 dominant and non-dominant leg during COD exercise.

236 Due to cost, tissue samples from three out of the five well-trained male team sports players (age: 27 $237 \pm 2$ yrs; height: $1.7 \pm 0.1 \mathrm{~m}$; weight: $73.5 \pm 11.1 \mathrm{~kg}$ ) were randomly taken forward for DNA methylome 238 analysis at baseline and immediately post and $24 \mathrm{hrs}$ post in both COD and ST trials. Tissue samples 239 were homogenized for 45 seconds at 6,000 rpm $\times 3$ (5 minutes on ice in between intervals) in lysis 240 buffer ( $180 \mu \mathrm{l}$ buffer ATL with $20 \mu$ l proteinase K) provided in the DNeasy spin column kit (Qiagen, UK) 241 using a Roche Magnalyser instrument and homogenization tubes containing ceramic beads (Roche, 
242 UK). Cells were lysed in $180 \mu$ I PBS containing $20 \mu$ proteinase $K$. The DNA was then bisulfite converted

243 using the EZ DNA Methylation Kit (Zymo Research, CA, United States) as per manufacturer's 244 instructions.

247 All DNA methylation experiments were performed in accordance with Illumina manufacturer 248 instructions for the Infinium Methylation EPIC BeadChip Array. Methods for the amplification, 249 fragmentation, precipitation and resuspension of amplified DNA, hybridisation to EPIC beadchip, 250 extension and staining of the bisulfite converted DNA (BCD) can be found in detail in our open access 251 methods paper ${ }^{3}$. EPIC BeadChips were imaged using the Illumina iScan System (Illumina, United 252 States). DNA methylation analysis, CpG enrichment analysis (GO and KEGG pathways), differentially modified region analysis and Self Organising Map (SOM) profiling

256 Following DNA methylation quantification via MethylationEPIC BeadChip array, raw .IDAT files were 257 processed using Partek Genomics Suite V.7 (Partek Inc. Missouri, USA) and annotated using the 258 MethylationEPIC_v-1-0_B4 manifest file. We first checked the average detection p-values. The mean 259 detection p-value for all samples was 0.000141 , and the highest was 0.00024 (Suppl. Figure 1a), which 260 is well below the recommended 0.01 in the Oshlack workflow ${ }^{17}$. We also examined the raw intensities/signals of the probes, that demonstrated an average median methylated and unmethylated signal of over 11.5 (11.74) and the difference between the average median methylated and average median unmethylated signal was 0.28 , well below the recommended difference of less than $0.5^{17}$. Upon import of the data into Partek Genomics Suite we filtered out probes located in known single-nucleotide polymorphisms (SNPs) and any known cross-reactive probes using previously defined SNP and cross-reactive probe lists identified in earlier EPIC BeadChip 850K validation studies 18. Although the average detection $p$-value for each sample across all probes was on average very low (no higher than 0.00024 ) we also excluded any individual probes with a detection $p$-value that was above 0.01 as recommended previously ${ }^{17}$. Therefore, out of a total of 865,860 probes, removal of known SNPs, cross-reactive probes and those with a detection p-value above 0.01 resulted in a final list of 809,877 probes analysed. Following this, background normalisation was performed via

272 functional normalisation (with noob background correction), as previously described ${ }^{19}$. Following 273 functional normalisation, we also undertook quality control procedures via principle component 274 analysis (PCA), density plots by lines as well as box and whisker plots of the normalised data for all 275 samples (Suppl. Figures $\mathbf{1 b}, \mathbf{c}$, $\mathbf{d}$ respectively). We confirmed that no samples demonstrated large 
variation (variation defined as any sample above 2 standard deviations (SDs) - depicted by ellipsoids in the PCA plots (Suppl. Figure 1b) and/or demonstrating any differential distribution to other samples, depicted in the signal frequency by lines plots (Suppl. Figure 1c)). Therefore, no outlier samples were detected. We used participants baseline samples from the COD trial only for array analysis, as these were resting biopsies from the same participant under the same conditions before each trial. We also confirmed that baseline samples were not different between COD and ST trials by running a participants COD and ST trial baseline samples and demonstrating that the PCA showed very little variation between the baseline samples taken before the COD trial and the baseline sample taken before the ST trial (Suppl. Figure 1c- Samples highlighted with an arrow). Following normalisation and quality control procedures, we undertook differentially methylated position (DMP) analysis by converting $\beta$-values to $M$-values $(M-$ value $=\log 2(\beta /(1-\beta))$, as $M$-values show distributions that are more statistically valid for the differential analysis of methylation levels ${ }^{20}$. We performed a 2-way ANOVA for 'Trial' (ST vs. COD) $x$ time (baseline, post, $24 \mathrm{~h}$ ) with planned contrasts of: 1) COD Post vs. ST post, 2) COD $24 \mathrm{~h}$ vs ST $24 \mathrm{~h}$ ). We also explored the ANOVA main effects for both 'Trial' to investigate the effect of trial alone (effect of COD vs. ST across time), and 'Time' (baseline, post and $24 \mathrm{~h}$ ) with planned contrasts (post vs. baseline, baseline vs $24 \mathrm{~h}$, post vs. $24 \mathrm{~h}$ ) to investigate the effect of time alone (exercise effect alone over time in both COD and ST groups). Any differentially methylated $\mathrm{CpG}$ position (DMP) with an adjusted $\mathrm{P}$ value of $\leq 0.01$ was deemed significant. We then undertook $\mathrm{CpG}$ enrichment analysis on these differentially methylated $\mathrm{CpG}$ lists within gene ontology (GO) and KEGG pathways ${ }^{21,22,23}$ using Partek Genomics Suite, Partek Pathway and Revigo (tree-maps for GO terms). Differentially methylated region (DMR) analysis, that identifies where several CpGs are consistently differentially methylated within a short chromosomal location/region, was undertaken using the Bioconductor package DMRcate (DOI: 10.18129/B9.bioc.DMRcate). Finally, in order to plot/visualise temporal changes in methylation across the time-course post exercise (COD vs. ST) we implemented Self Organising Map (SOM) profiling of the change in mean methylation within each condition using Partek Genomics Suite.

\section{Comparative transcriptome analysis}

304 We overlapped the significant differential sprint methylome data from the present study with 305 significantly expressed sprint transcriptomes ${ }^{13}$ and also with a meta-analysis of acute aerobic exercise 306 transcriptomes ${ }^{14}$. Venn diagram analysis was used to generate gene lists of overlap between 307 differential methylome and transcriptome data. We also used this approach to confirm the genes that 308 were identified to be hypomethylated in the present study and upregulated in the sprint 309 transcriptome ${ }^{13}$, that were also upregulated in the aerobic exercise transcriptome meta-analyses ${ }^{14}$. 
310 As well as genes that were hypermethylated in the present study and down-regulated in the

311 transcriptome data sets. Metamex.eu ${ }^{14}$ was used to confirm the genes logfold change and significance

312 (with an FDR of $\mathrm{p} \leq 0.05$ ) in the meta-analysis transcriptome data. In particular, we selected the criteria

313 of healthy skeletal muscle up to $24 \mathrm{hrs}$ post exercise in order to match to the design of the present

314 study. To generate a list of genes that were significantly differentially methylated in the present study

315 and significantly regulated at the expression level in transcriptome meta-analysis after acute aerobic

316 exercise (but not identified in the sprint transcriptome) ${ }^{13}$ we conducted new analysis of acute aerobic

317 exercise transcriptome datasets from the meta-analysis ${ }^{14}$. Therefore we also analysed full significant

318 genes lists (adj. $p$ value of $\leq 0.05$ ) that were up- or down-regulated in transcriptome meta-analysis

319 after acute aerobic exercise and overlapped these lists with our most significant methylated DMP

320 analysis. New correlation analysis was performed on the non-publicly available acute aerobic meta-

321 analysis data ${ }^{14}$ for the genes identified in the present manuscript at the methylome, comparative

322 transcriptome and targeted gene level (RT-PCR below) to be significantly altered (including VEGFA,

323 NR4A1, NR4A3 and PGC1- $\alpha$ ).

RNA isolation, primer design \& gene expression analysis

326 Skeletal muscle tissue muscle from the same samples as the DNA methylome analysis across both

327 trials (ST and COD) at baseline, $3 \mathrm{~h}$ and $24 \mathrm{~h}$ post was homogenised in tubes containing ceramic beads

328 (MagNA Lyser Green Beads, Roche, Germany) and $1 \mathrm{ml}$ Tri-Reagent (Invitrogen, Loughborough, UK)

329 for 45 seconds at $6,000 \mathrm{rpm} \times 3$ (and placed on ice for 5 minutes at the end of each 45 second

330 homogenization) using a Roche Magnalyser instrument (Roche, Germany). RNA was then isolated as

331 per Invitrogen's manufacturer's instructions for Tri-reagent. Then a one-step RT-PCR reaction (reverse

332 transcription and PCR) was performed using QuantiFast SYBR Green RT-PCR one-step kits on a

333 Rotorgene $3000 \mathrm{Q}$. Each reaction was setup as follows; $4.75 \mu \mathrm{l}$ experimental sample $(7.36 \mathrm{ng} / \mu \mathrm{l}$

334 totalling $35 \mathrm{ng}$ per reaction), $0.075 \mu \mathrm{l}$ of both forward and reverse primer of the gene of interest (100

$335 \mu \mathrm{M}$ stock suspension), $0.1 \mu \mathrm{l}$ of QuantiFast RT Mix (Qiagen, Manchester, UK) and $5 \mu$ l of QuantiFast

336 SYBR Green RT-PCR Master Mix (Qiagen, Manchester, UK). Reverse transcription was initiated with a

337 hold at $50^{\circ} \mathrm{C}$ for 10 minutes (cDNA synthesis) and a 5-minute hold at $95^{\circ} \mathrm{C}$ (transcriptase inactivation

338 and initial denaturation), before $40-50$ PCR cycles of; $95^{\circ} \mathrm{C}$ for $10 \mathrm{sec}$ (denaturation) followed by $60^{\circ} \mathrm{C}$

339 for $30 \mathrm{sec}$ (annealing and extension). Primer sequences for genes of interest and reference genes

340 were. VEgFA Fwd ACGgtCCCTCTTGgAATtGG, Rvse CTAATCTTCCGGgCTCGgTG; NR4A1 Fwd

341 GgTGACCCCACGATTTGTCT, Rvse GGCTTATTTACAGCACGgCG; NR4A3 Fwd

342 GacgtcgaAaccGatgtcag, Rvse tTtggaAggCAgAcGacctc, PGC1- $\alpha$ Fwd

343 TGCTAAACGACTCCGAGAA, Rvse TGCAAAGTTCCCTCTCTGCT; $\quad$ RPL13a $\quad$ Fwd 
GgCtAAACAGGTACTGCTGGG, Rvse AgGAAAGCCAGGTACTTCAACTT. All primers were designed to yield products that included the majority of transcript variants for each gene as an impression of total changes in the gene of interests' expression levels. All genes demonstrated no unintended gene targets via BLAST search and yielded a single peak after melt curve analysis conducted after the PCR step above. All relative gene expression was quantified using the comparative $\mathrm{Ct}\left({ }^{\Delta \Delta} \mathrm{Ct}\right)$ method ${ }^{24}$. The baseline sample for each participant and their own reference gene sample was used as the calibrator conditions. The average, standard deviation and variations in Ct value for the RPL13a reference gene demonstrated low variation across all samples (mean \pm SD, $21.04 \pm 1.49,7.1 \%$ variation) for the analysis. The average PCR efficiencies for VEGFA, NR4A1, NR4A3 and PGC1- $\alpha$ were comparable $190 \pm$ $5.2,89.2 \pm 4.2,91.3 \pm 6.4 \%, 92.5 \pm 1.9$ variation) with the reference gene RPL13a (89.1 $\pm 4.8 \%)$. Statistical analysis genes were performed using a 2-way ANOVA with fisher post-hoc comparisons at the level of $P$ value of $\leq 0.05$ using Graphpad.

Results

Change of direction exercise elicits increased loading compared with ST exercise

360 Change of direction exercise increased mean movement loading (GPS Playerload $\left.{ }^{\mathrm{TM}}\right)(3.35 \pm 0.07$ vs.

$3612.93 \pm 0.08$ au; $p \leq 0.001$ ), physiological loading (heart rate post exercise) (159 \pm 16.3 vs. $137 \pm 13.5$

$362 \mathrm{bpm} ; p \leq 0.001)$, metabolic loading (lactate 5 minutes post exercise) $(8.45 \pm 1.7 \mathrm{vs} .1 .69 \pm 0.68 \mathrm{mmol} / \mathrm{L}$

$363 \mathrm{p} \leq 0.05$ ), subjective (differential RPE) peripheral loading ( $47 \pm 24$ vs. $10 \pm 6 ; p \leq 0.001$ ) and central loading (62 \pm 29 vs. $10 \pm 6 ; p \leq 0.001$ ) versus straight line exercise.

366 Exercise alone evokes a hypermethylated response immediately post exercise followed by 367 hypomethylated signature by $24 \mathrm{~h}$, particularly in MAPK, AMPK and Axon Guidance pathways.

368 A 1-way ANOVA for 'time' (baseline, immediately post, $24 \mathrm{~h}$ after exercise), that analyses the main 369 effect of sprint exercise alone over time across both experimental trials (COD and ST trials) identified 370 a list of 7,612 significantly differentially methylated CpG positions (DMP's) (Suppl. File 1a). Within this 371 'exercise alone' list, 1,076 DMPs were promoter associated with 1,651 located in CpG islands. Planned 372 contrasts within this ANOVA for baseline vs. post exercise time points, also identified 4,952 significant 373 DMPs (Suppl. File 1b). Where a larger number of DMPs were hypermethylated post exercise versus 374 baseline, with 3287 hyper- and 1665 hypo-methylated. At 24 hrs ( $24 \mathrm{~h}$ vs. baseline contrast) there was 375 6,638 DMPs (Suppl. File 1c), that demonstrated a shift towards a hypomethylated profile at $24 \mathrm{~h}$, with 3763,617 hypermethylated vs. 3,021 hypomethylated DMPs. Furthermore, this shift was confirmed in the $37724 \mathrm{~h}$ vs. post contrast, that identified a DMP list of 13,139 CpGs (Suppl. File 1d), with a predominately 
378

hypomethylated profile, of 8,413 hypomethylated vs. 4726 hypermethylated DMPs. These data therefore suggest that immediately post exercise (across both experimental trials, COD and ST) DMPS favoured hypermethylation, however, after that point and up to $24 \mathrm{hrs}$ there was shift towards a hypomethylated profile. This observation can be visualised as a hierarchical heat map in Figure 1a. Finally, SOM temporal analysis of the main effect for 'time' 7,612 DMPs also confirmed the above analysis and suggested that the largest group of DMPs (e.g. 3,052 CpG's highlighted in red and located in SOM profile; Figure 1b) were hypermethylated immediately post exercise, with the same DMPs demonstrated a shift to a hypomethylated profile $24 \mathrm{~h}$ after exercise.

$1 a$

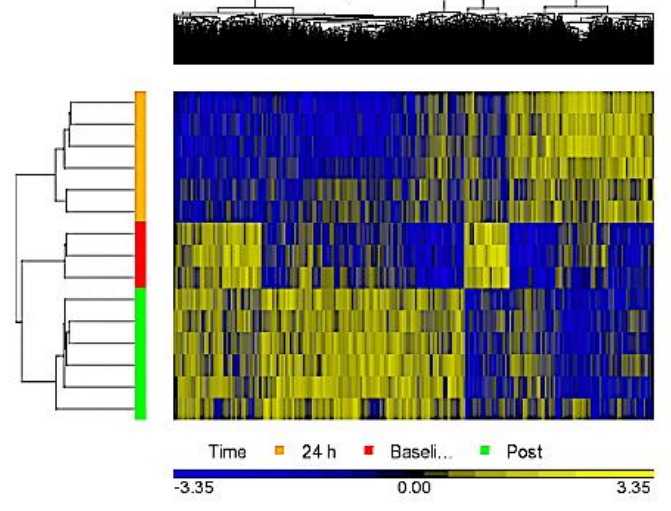

$1 b$

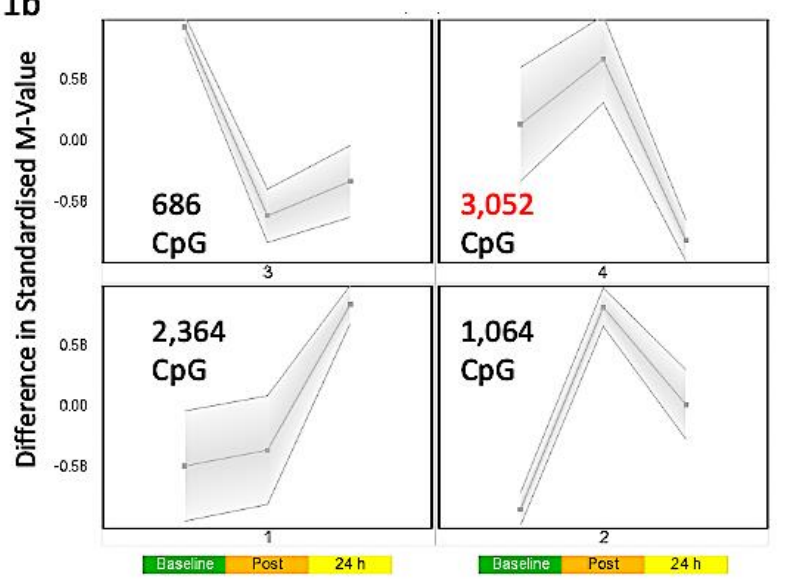

Figure 1. a. Hierarchical heat map for analysis of DMPs over exercise time in both trials (Red - Baseline, Post- Green, $24 \mathrm{~h}$ Orange). b. SOM temporal analysis of DMPs over exercise time in both trials.

We next wished to identify which gene ontology (GO) terms and KEGG pathways were significantly enriched over the time-course of baseline, post and 24 following exercise (across both experimental trials (COD and ST). Indeed, GO enrichment analysis of the post vs. baseline contrast (Suppl. File 1e) identified that there was significant hypermethylation enrichment of DMPs in top 5 GO terms: 1) Neuron Part, 2) developmental process, 3) cellular component organisation, 4) regulation of neuron projection development and, 5) cellular component organisation or biogenesis (Suppl. File $\mathbf{1 f}, \mathbf{g}, \mathbf{h}, \mathbf{i}, \mathbf{j}$ respectively). For the $24 \mathrm{~h}$ versus baseline contrast (Suppl. File 1k) DMP hyper/hypomethylation was more balanced in top GO 5 terms: 1) Protein binding, 2) binding, 3) organelle, 4) intracellular part, and 5) intracellular organelle (Suppl. File $\mathbf{1 l}, \mathbf{m}, \mathbf{n}, \mathbf{0}, \mathbf{p}$ ). Then at $24 \mathrm{~h}$ compared with post exercise (Suppl. File 1q) top 5 GO terms enriched predominantly hypomethylated were: 1) Developmental process, 2) anatomical structure development, 3) anatomical structure morphogenesis, 4) regulation of signaling and, 5) regulation of cell communication (Suppl. File $\mathbf{1} \mathbf{r}, \mathbf{s}, \mathbf{t}, \mathbf{u}, \mathbf{v}$ ). 
For KEGG pathway analysis, in post vs. baseline contrasts (Suppl. File 2a) there was significant enrichment of hypermethylation of DMPs in top 5 KEGG pathways of: 1) MAPK signaling pathway, 2) axon guidance, 3) human papillomarvirus infection, 4) small cell lung cancer and, 5) insulin secretion (Suppl. Files 2b, c, d, e, f). At $24 \mathrm{~h}$ vs. baseline contrast (Suppl. Files $\mathbf{2 g}$ ) top 5 enriched KEGG pathways were: 1) MAPK signaling pathway, 2) AMPK signaling pathway, 3) lysine degradation, 4) phosphonate and phosphate metabolism and, 5) aldosterone synthesis and secretion (Suppl. Files, 2h, i, j, k, l). At $24 \mathrm{~h}$ vs. post exercise (Suppl. Files $\mathbf{2 m}$ ) hypomethylated profile enriched in top 5 KEGG pathways: 1 ) axon guidance, 2) cGMP-PKG signaling pathway, 3) focal adhesion, 4) rap1 signaling, and 5) adrenergic signaling in cardiomyocytes' (Suppl. Files, 2n, o, p, q, r).

412

413 Finally, DMR analysis of the post vs. baseline (Suppl. File 2s), $24 \mathrm{~h}$ vs. baseline (Suppl. File 2t) and 24

$414 \mathrm{~h}$ vs. post contrasts (Suppl. File $\mathbf{2 u}$ ) identified several regions located in or close to annotated genes with enriched (multiple CpGs) differential methylation. Including enriched hypermethylation of genes: RBMXL1, ALDH3A1, EVA1A, MiR3928, RNF185, ANAPC10 and ABCE1 (post vs. baseline) and c12orf42, HSPD1, HSPE1, SALL1, CCNDN2 (24 h vs. baseline) and enriched hypomethylation in genes: ZIC1, ZIC4, TBX15, NAV2 and OXT (24h vs. post).

Change of direction exercise evokes a greater hypomethylated signature in protein binding and axon guidance pathways compared with straight line exercise

Analysing the main effect for 'Trial' (COD vs. ST sprint exercise across all time points) identified 13,218 significant DMPs (Suppl. File 3a) favouring hypomethylation over hypermethylation $(8,627$ hypo- vs 4654 hyper-methylated). Undertaking hierarchical clustering of these DMPs enabled visualisation of the predominance in hypomethylation in COD versus the ST sprint exercise trials (Figure 2a). A large number of DMPs 4,221 (out of 13,218) were promoter associated, with 5,304 (out of 13,218 ) located in CpG islands. Undertaking gene ontology (GO) pathway enrichment within this 13,218 DMP list (Suppl. File 3b). The top $5 \mathrm{GO}$ terms enriched for hypomethylation in COD vs. ST sprint exercise were: 1) intracellular part, 2) protein binding, 3) binding, 4) organelle and, 5) intracellular organelle part (Suppl. File 3c, d, e, f, g), predominantly within overarching 'cellular component' (Figure 2b) and 'molecular function' gene ontologies (Figure 2c). The top KEGG pathways (Suppl. File 3h) predominantly hypomethylated were: 1) Axon guidance (Figure 2d), 2) cell cycle, 3) endocytosis, 4) pathways in cancer and, 5) spliceosome (Suppl. Files $\mathbf{3} \mathbf{i}, \mathbf{j}, \mathbf{k}, \mathbf{I}, \mathbf{m}$ respectively). DMR analysis also identified that the genes: E2F3, BRD2, MUS81 and CFL1 had enriched hypomethylation of multiple 
437

438

439

440

441

442

443

444

445

446

447

448

449

450

451

452

453

454

455

456

457

458

459

460

461

462

463

464

465

466

467

468

469

470

471

472

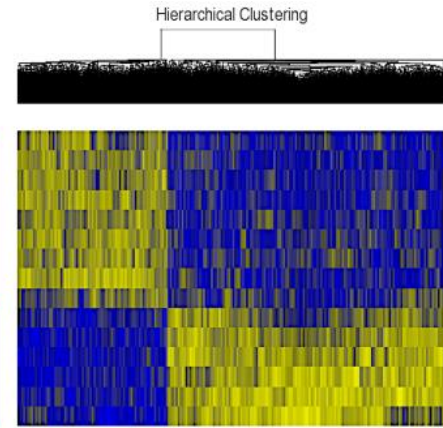

" Thial Change of Direction (COD)

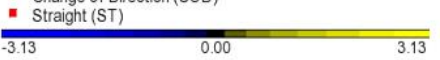

2c

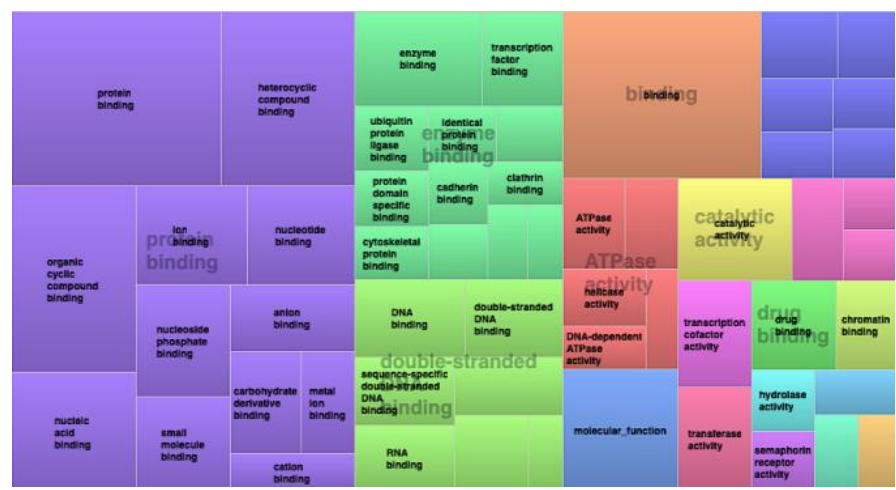

5

Figure 2. a. Hierarchical heat map for analysis of DMPs in COD vs. ST trials across all time. b. DMP enrichment TreeMap of overarching Gene Ontologies (GO) term 'cellular component' in COD vs. ST trials. Demonstrating top enriched GO terms of 'intracellular part', 'organelle' and 'intracellular organelle part'. c. DMP enrichment TreeMap of overarching Gene Ontologies (GO) 'molecular function'. Demonstrating top enriched GO terms of 'protein binding' and 'binding'. d. Illustration of the top KEGG pathway 'axon guidance' demonstrating predominantly hypomethylation (blue) in COD vs. ST sprint trials. Note, the most significant (lowest p-value) DMP for each gene is used to colour this pathway image. Therefore, this is not always accurate where multiple DMPs occur for a single gene and the image is therefore only a visual representation of the overarching methylation profile in this pathway. Full and accurate DMP lists for the axon guidance pathway in these conditions can be found in (Suppl. File 3i).

Change of direction exercise evokes the largest hypomethylated signature immediately post exercise compared with straight line exercise, particularly in protein binding, axon guidance and insulin related pathways

A 2-Way ANOVA for 'Trial' $x$ 'Time' interaction, that demonstrates a difference in methylation between COD and ST sprint exercise immediately post and 24 hrs post exercise, identified 7,844 significant DMPs (Suppl. File 4a). 1,349 DMPs were promoter associated with 1,846 DMPs within CpG islands. Contrasts for COD post vs. ST post identified 6,489 significant DMPs (Suppl. File. 4b). Where 
473 hypomethylation was predominant post exercise in COD vs. ST post exercise, with 4,472

474 hypomethylated and 2,017 hypermethylated DMPs (Figure 3a). At 24 hrs (COD 24 h vs. ST 24 h) there 475 were 6,647 significant DMPs (Suppl. File 4c), where the shift in favouring hypomethylation post 476 exercise was now more balanced with hypermethylation at $24 \mathrm{~h}$, yet still with a larger total of 477 hypomethylated $(3,649)$ compared with hypermethylated $(2,953)$ DMPs (Figure 3b). Furthermore, 478 SOM analysis of mean temporal changes generated over time from the mean of each condition using 479 the interaction Trial $x$ Time (7,844 DMP) list demonstrated the following temporal profiles depicted in 480 Figure $\mathbf{3 c}$ for COD exercise and Figure $\mathbf{3 d}$ for ST exercise. Indeed, these profiles confirmed that the 481 majority of DMPs in the COD trial demonstrated a hypomethylated compared with hypermethylated 482 profile particularly in the immediately post exercise timepoint.
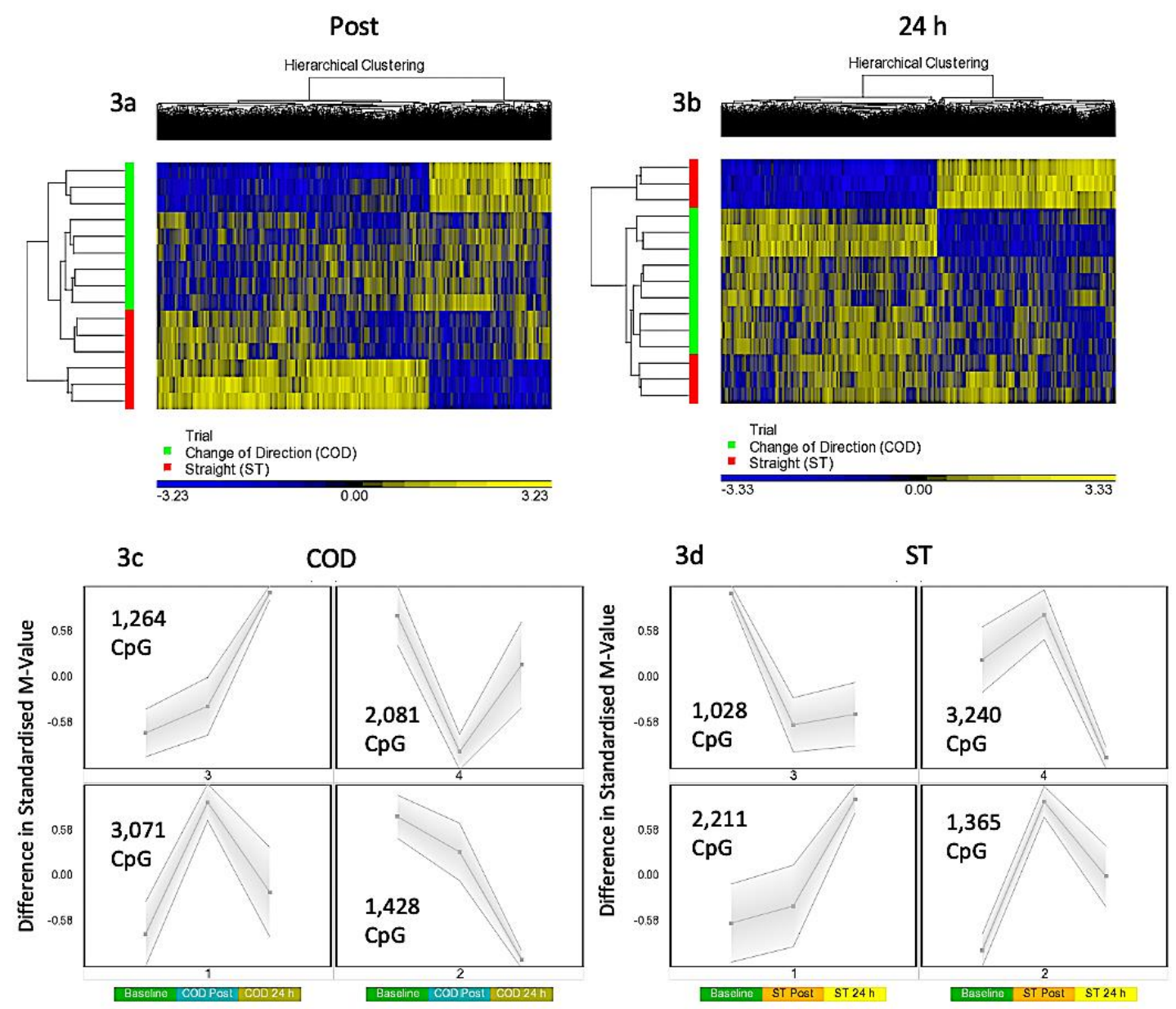

483

484

Figure 3. a. Hierarchical heat map for analysis of DMPs in COD vs. ST trials immediately post exercise. b. Hierarchical heat map for analysis of DMPs in COD vs. ST trials $24 \mathrm{~h}$ post exercise c. SOM temporal analysis of DMPs over exercise time in COD exercise trial at baseline, immediately post and $24 \mathrm{hr}$ post exercise. d. SOM temporal analysis of DMPs over exercise time in ST exercise trial at baseline, immediately post and $24 \mathrm{hr}$ post exercise. In gene ontology (GO) analysis of the 2 Way ANOVA 'Trial' $x$ 'Time' interaction (7,844 DMP list; Suppl. File 4d), identified overarching GO terms: Biological process, cellular component, and molecular 
491 function, as well as the top GO term protein binding (within molecular function) (Suppl. File 4e) that

492 demonstrated more CpG's hypomethylated compared with hypermethylated (2,276 hypomethylated

493 versus 1,386 hypermethylated). The same trend was observed after KEGG pathway analysis (Suppl.

494 File $\mathbf{4 f}$ ) where the top differentially enriched pathway was 'axon guidance' (Suppl. File $\mathbf{4 g}$ ) with more

495 CpG's hypomethylated in COD post vs. ST post exercise (61 CpG hypomethylated, 44 CpG

496 hypermethylated).

497

498 More specifically post exercise in COD vs. ST trials, gene ontology (GO) analysis of the differentially 499 methylated CpG's within this contrast (6,489 DMP list above; Suppl. File 4h) demonstrated the most 500 significantly enriched top 5 GO terms as: 1) Protein binding (Suppl. File 4i), 2) binding, 3) positive 501 regulation of cellular metabolic process, 4) positive regulation of metabolic process, and 5) positive 502 regulation of macromolecule metabolic process (Suppl. Files $\mathbf{4 j}, \mathbf{k}, \mathbf{I}, \mathbf{m}$ ) that all demonstrated more $503 \mathrm{CpG}$ 's that were hypomethylated vs. hypermethylated in these GO terms. By $24 \mathrm{hrs}$ in the 6,647 DMP 504 list (contrast COD $24 \mathrm{~h}$ vs. ST $24 \mathrm{~h}$ ) there was a shift back to a more balanced hypo/hypermethylated ratio in the top 5 GO terms (Suppl. File 4n) for: 1) protein binding (Suppl. File 40), 2) binding, 3) regulation of signaling, 5) regulation of cell communication, and 5) anatomical structure morphogenesis; Suppl. File 4p, q, r, s).

508

The same trend was observed after KEGG pathway analysis (Suppl. File 4t) where the top differentially enriched hypomethylated pathways were: 1) Insulin resistance, 2) endocytosis, 3) axon guidance, 4) MAPK signalling pathway and, 5) insulin signaling pathway in the COD post vs. ST post contrast (Suppl.

512 Files $4 \mathbf{u}, \mathbf{v}, \mathbf{w}, \mathbf{x}, \mathbf{y}$ ). In the $24 \mathrm{~h}$ vs. $24 \mathrm{~h}$ ST trial contrast KEGG pathway analysis (Suppl. File 5a) with 513 more balanced hypo/hypermethylation ratio: 1) ErbB signaling, 2) Non-small cell lung cancer, 3) axon 514 guidance, 4) proteoglycans in cancer, and 5) glioma (Suppl. Files $\mathbf{5 b}, \mathbf{c}, \mathbf{d}, \mathbf{e}, \mathbf{f})$. In DMR analysis COD post 515 vs. ST post (Suppl. File 5g), identified enriched hypomethylation in genes: CIITA, PRR5, WDR46/PFDN6, 516 MAGI2, RNF167 and DGKZ. In COD $24 \mathrm{~h}$ vs. ST $24 \mathrm{~h}$ DMR analysis suggested genes COL1A1 and CHID1 possessed enriched hypermethylation (Suppl. File $5 \mathbf{h}$ ).

519 Comparative methylome and transcriptome analyses identifies inverse relationships in DNA 520 methylation and gene expression: NR4A1 is hypomethylated and associated with increased gene expression after acute exercise.

523 In order to identify genes that were differentially methylated and also altered at the gene expression 524 level after exercise between COD and ST sprint exercise trials, we first overlapped the present studies 
methylome data (significant DMPs main effect for 'time' 7,612 CpG list, main effect for 'trial' 13,281

526 Cpg list and the interaction 'time' $x$ 'trial' 7,844 CpG list) with recent published transcriptome data

527 sets following acute sprint exercise ${ }^{13}$. In this sprint transcriptome, the authors identified 879 genes

528 that were significantly differentially expressed post-exercise (471 upregulated / 408 downregulated -

529 List in Suppl. File 6a). Out of these 879 genes that had altered gene expression across the sprint

530 transcriptome, there was large overlap of genes (49\% or 431 genes) that were also differentially

531 methylated in at least one of the significant DMP analyses in the present study (Figure 4a, Suppl. File

$5326 \mathbf{b}$ for gene list). To determine genes that were frequently occurring DMPs in COD vs. ST sprint exercise

533 trials, that mapped through to significant differential gene expression in the transcriptome analysis,

534 using Venn diagram analysis we first identified that out of these 431 genes, 61 were shared by all

535 methylation analyses in the present study (Figure 4a, Suppl. File 6c). Eighteen of the genes that were

536 upregulated, also displayed hypomethylation (on 27 DMPs) immediately post exercise in COD vs. ST

537 sprint exercise condition (Suppl. File 6d). Fifteen of the genes, that were downregulated were also

538 hypermethylated (24 DMPs) immediately post exercise in COD vs. ST conditions (Suppl. File 6e).

539 Fifteen genes were also upregulated and hypomethylated (21 DMPs) 24 hrs post exercise (Suppl. File

540 6f) and twenty-one of these genes were downregulated and hypermethylated (30 DMPs) 24 hrs post

541 exercise (Suppl. File 6g). Those genes with the same trend (down and hypermethylated) at both time

542 points (post and 24hrs) in COD vs. ST exercise trials, included a final list of 13 genes (containing 28

543 DMPs) including: ATP2C1, CCDC88C, FAM111A, GAB1, HMCN1, LRRTM4, LTBP1, MAGI1, MYH10,

544 MYO10, NEDD9, NIPAL3 and RHOBTB1 (Suppl. File 6h). With 10 genes (containing 22 DMPs) that were

545 upregulated and hypomethylated at both post and $24 \mathrm{hrs}$ in COD vs. ST sprint exercise trials, including

546 genes: ADM, DUSP1, IRS2, MAP2K3, PDE2A, PDE4E, PLXNA2, POR, PPP1R15A and VEGFA (depicted

547 Figure $\mathbf{4 b}$, Suppl. File $6 \mathrm{i})$. The majority of these hypomethylated and upregulated genes were

548 associated with the MAPK pathway, together with the canonical angiogenesis gene, VEGFA (see

549 additional DMR data for VEGFA below).

551 In addition, we looked to validate if the genes identified in the present study's methylome and recent

552 sprint transcriptome were also regulated in the most comprehensive set of acute aerobic

553 transcriptomes to date ${ }^{14}$. Indeed, the vast majority of the 13 genes found to be

554 hypermethylated/down-regulated and 10 genes hypomethylated/up-regulated in the present study's

555 methylome and sprint transcriptome ${ }^{13}$ were all significantly regulated (FDR < 0.05; Metamex.eu) at

556 the gene expression level (except 2 genes; DUSP1 and NEDD9) in healthy skeletal muscle up to 24

557 hours after acute aerobic exercise in the transcriptome meta-analysis ${ }^{14}$. Most importantly, in the

558 meta-analyses' acute aerobic transcriptome data all these genes were regulated in same direction as 
identified above. This comparative methylome and transcriptome analysis therefore confirmed that there was an inverse relationship between these genes' DNA methylation profile and their gene expression after acute exercise. Furthermore, re-analysing the full gene lists in the acute aerobic exercise transcriptomes meta-analysis data set ${ }^{14}$, we identified an additional 10 genes that were significantly up/down-regulated in the transcriptome meta-analyses (but not identified in the sprint transcriptome), that also demonstrated significantly altered methylation across all analyses in the present study (Figure 4a). These included: NR4A1, ADAMTS9, DLGAP4, GNAL, NPHP1, PRKAG2, SLC15A4, SLFN11, SYNJ2, WDR81 (Figure 4b, Suppl. File 7a,b). Notably, within this list, NR4A1 is a nuclear receptor and family member to NR4A3 that has been identified as one of the most exerciseand inactivity-responsive genes across all 66 published transcriptome data sets ${ }^{14}$. NR4A3 has a role in mediating the metabolic responses to exercise-like stimuli in vitro ${ }^{14}$. NR4A1 is also within KEGG pathway of 'MAPK signalling' and GO term 'protein binding' identified above as enriched hypomethylated pathways in COD vs. ST exercise. We identify that NR4A1, was hypomethylated in its promoter located in a CpG island close to its transcription start site TSS200 (cg11666140) in COD vs. ST exercise trials (Figure 4c, Suppl. File 7a), with another s-shelf associated CpG also identified (cg20661548) to be hypomethylated immediately post exercise in COD vs. ST trials (Suppl. File 7b). Further analysis shows that, alongside NR4A3, NR4A1 gene expression was also significantly upregulated ( $0.94 \mathrm{log}$ fold) in exercise-transcriptome meta-analyses ${ }^{14}$ and within the top ten genes upregulated in the sprint transcriptome ${ }^{13}$. NR4A1 expression was also ranked $2^{\text {nd }}$ highest in its significant positive correlation with NR4A3 $\left(r=0.72\right.$, metamex.eu $\left.{ }^{14}\right)$ across the 66 published exercise and inactivity transcriptome data sets (metamex.eu ${ }^{14}$ ), with new analysis of NR4A1 and NR4A3 gene within meta-analyses transcriptomes of healthy young adult up to $24 \mathrm{hrs}$ (to match the present study design) also suggesting a highly significant positive correlation ( $r=0.6, F D R=0.007$; Figure $4 d$ ). Indeed, we were able to observe an elevation in NR4A1 at $3 \mathrm{hrs}$ and $24 \mathrm{hrs}$ post exercise in COD vs. ST trails,

583 but this was not statistically significant (Figure 4e). Importantly however, we observed significant 584 increases $(p \leq 0.05)$ of a large magnitude in NR4A3 gene expression $(165.2$ vs 6.8 -fold, $p \leq 0.05)$ in COD vs. ST trial at 3 hours post exercise (Figure $\mathbf{4 f}$ ). Therefore, the comparative analysis of our present methylome data and its overlap across recent sprint transcriptome ${ }^{13}$ and meta-analysis transcriptomes after acute aerobic exercise ${ }^{14}$, provides evidence to suggest that there is an inverse relationship between the above genes methylation and gene expression profile. Also identifying for the first time, that NR4A1 is an epigenetically regulated exercise gene and that NR4A3 is hugely upregulated (165-fold) as a consequence of performing high intensity change of direction compared with straight line sprint exercise.

592 
593 VEGFA has enriched hypomethylation in its promoter and is strongly associated with increased gene 594 expression across exercise transcriptomes

595 We identified above that VEGFA was hypomethylated in the present study and gene expression 596 increased across sprint and acute aerobic exercise meta-analysis transcriptomes. DMR analysis also 597 identified enrichment of hypomethylation (3 DMPs) on the VEGFA gene (DMPs- cg01116220, 598 cg04629501 and cg21099624- Figure 4c, Suppl. File 8a) in COD vs. ST exercise trails. All these DMPs 599 were located amongst a CpG island within a 1093 base pair locus. Two of these DMPs were also within 600 VEGFAs promoter region close to its transcription start site (TSS) and $1^{\text {st }}$ exon (cg21099624 - TSS200, 601 cg01116220 - Exon 1, Figure 4a, Suppl. File 8b). Overall, suggesting that this gene has enriched 602 hypomethylation in its promoter in COD compared with ST sprint exercise trials. Finally, as mentioned 603 above, VEGFA expression was significantly increased in the sprint transcriptome data set ${ }^{13}$, but was 604 also one of the most upregulated genes (0.96 log fold change; metamex.eu) in the transcriptome 605 meta-analysis for acute aerobic exercise in healthy males up to $24 \mathrm{hrs}$ after exercise ${ }^{14}$. VEGFA was 606 also ranked top $\left(1^{\text {st }}\right)$ most significantly correlated gene at the expression level with the canonical 607 exercise/metabolic regulators PGC1- $\alpha\left(r=0.84\right.$, metamex.eu) and NR4A3 $\left(r=0.74\right.$, metamex.eu $\left.{ }^{14}\right)$ 608 that was the most significantly upregulated across all modes of exercise ${ }^{14}$ and identified as 609 hypomethylated in its promoter in the present study. Correlation analysis of VEGFA with PGC1- $\alpha$ and 610 NR4A3 genes within meta-analyses transcriptomes of healthy young adults immediately post and up 611 to $24 \mathrm{hrs}$ (to match the current study design) post-acute aerobic exercise also suggested a significant 612 correlation between VEGFA and PGC1- $\alpha(r=0.5$, FDR $=0.031$; Figure 4g) and NR4A3 $(r=0.67$, FDR $=$ 613 0.002; Figure 4h). Further, VEGFA was significantly positively correlated with NR4A1 ( $r=0.58$, 614 metamex.eu ${ }^{14}$ ) across all exercise transcriptomes and also after analysis of acute-aerobic exercise 615 transcriptomes in healthy young adults $(r=0.54$, FDR $=0.02$; Figure 4i). Importantly, we identified 616 VEGFA to possess enriched hypomethylated in its promoter in the current study. Interrogation of aged 617 skeletal muscle tissue methylome in our recent studies, also suggests one of the same VEGFA CPG 618 sites (cg04629501) is oppositely regulated (hypermethylated) with age ${ }^{25}$ compared with exercise 619 (hypomethylated) in the present study. Therefore, VEGFA is hypomethylated in its promoter and is 620 associated with its own increased gene expression (as well as highly correlated with PGC1- $\alpha$, NR4A1 621 and NR4A3 expression) in the post-acute aerobic exercise transcriptome. Finally, VEGFA gene 622 expression was elevated at 24 hours $(p=0.01)$ in COD vs. ST trials (Figure $4 \mathrm{~J})$. We also observed 623 significant increases $(p \leq 0.05)$ of a large magnitude in canonical metabolic gene, PGC1- $\alpha$ (11.6 vs. 3.8624 fold in COD vs. ST) in COD vs. ST trial at 3 hours post exercise (Figure 4K). Overall, for the first time this 625 study identifies VEGFA as an epigenetically regulated gene in the response to acute exercise. 

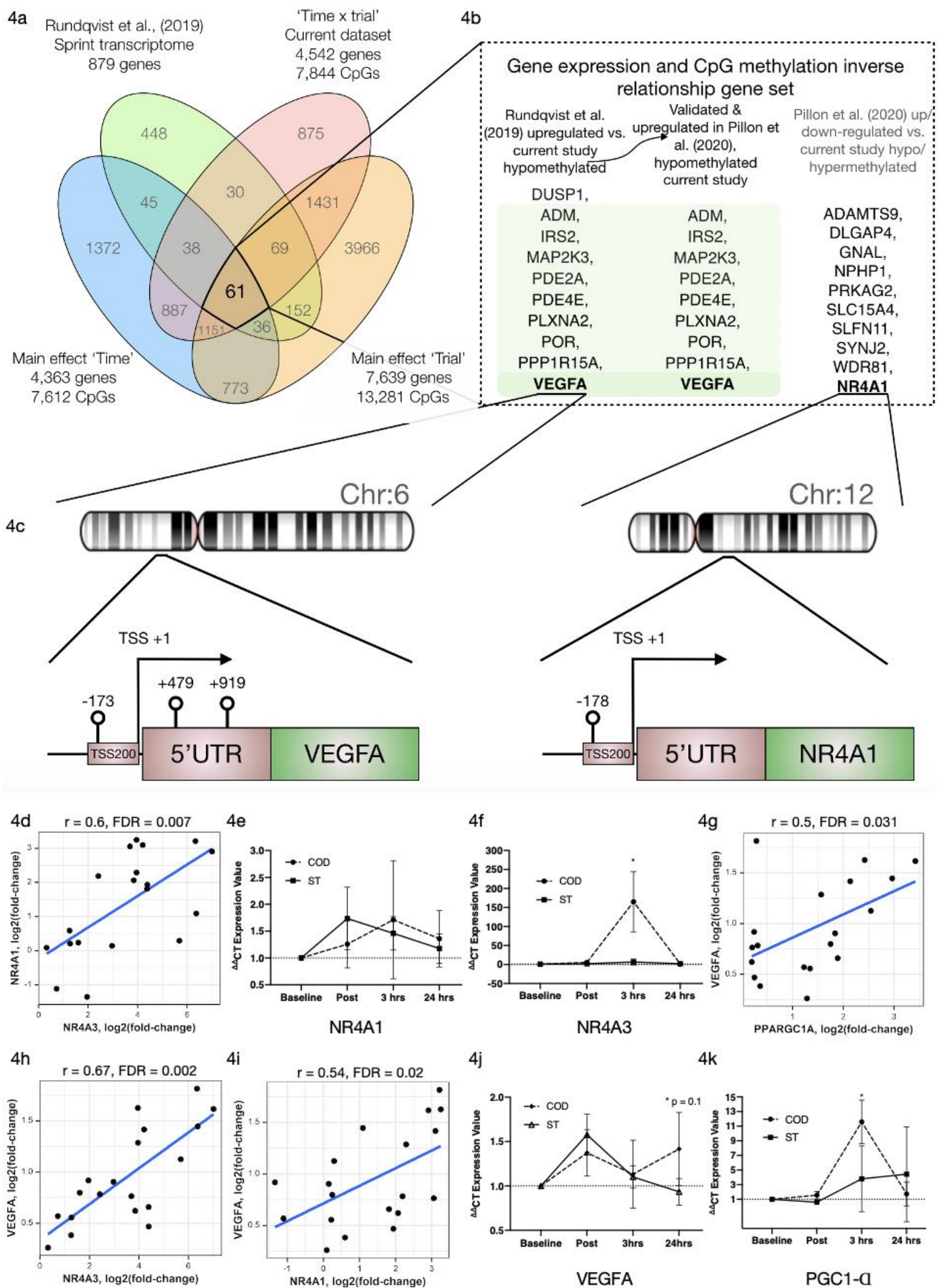

$4 \mathrm{j} \quad 4 \mathrm{k}$
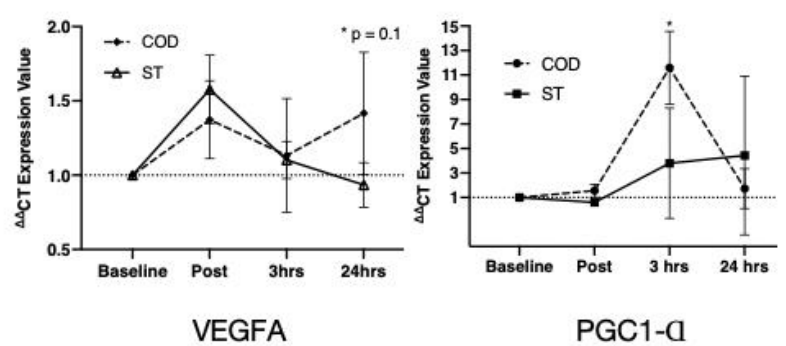

628 Figure 4. a. Venn diagram analyses of the overlap between the sprint transcriptome ${ }^{13}$ and differential methylome analysis in the present study. A total of 431 genes overlapped with the sprint transcriptome and were identified as significant DMPs in at least one of the methylome analyses in the present study. 61 genes were and up/down regulated in the sprint transcriptome and significantly differentially methylated across all methylome analyses in the present study. b. Genes 
632 (shaded in green) out of the 61 identified in figure 4a, that were significantly upregulated in the sprint transcriptome ${ }^{13}$,

633 upregulated in acute aerobic exercise transcriptome meta-analysis ${ }^{14}$ as well as hypomethylated in all methylome analyses

634 in the present study. Unshaded genes were upregulated in acute aerobic exercise transcriptome meta-analysis ${ }^{14}$ (but not

635 changed in the sprint transcriptome) and significantly hypo or hyper methylated in all methylome analyses in the present

636 study. d. Highly significant positive correlations in gene expression for NR4A1 vs. NR4A3 e. Gene expression of NR4A1 and $\mathbf{f}$.

637 NR4A3 at post, 3 hrs and 24 hrs after COD vs. ST exercise. Highly significant positive correlations in gene expression for $\mathbf{g}$.

638 VEGFA vs. PPARGC1A (PGC1- $\alpha$ ), h. VEGFA vs. NR4A3 and i. VEGFA vs. NR4A1 in young healthy adult skeletal muscle

639 immediately post up to 24 hours post exercise in new reanalysis of all acute aerobic transcriptomes meta-analyses ${ }^{14}$. Gene

640 expression for j. VEGFA and k. PGC1- $\alpha$ at post, 3 hrs and 24 hrs after COD vs. ST exercise. * $p \leq 0.05$ unless otherwise stated.

641

642 Discussion

643 The present study aimed to investigate the methylome and transcriptome immediately post and 24

644 hrs after acute exercise in two sport and exercise relevant sprint shuttle running protocols: 1) Change

645 of direction (COD) and, 2) straight line (ST) sprint exercise. We first demonstrated that while sprint

646 trials were matched for classically defined intensity measures (speed $\mathrm{x}$ distance) and the number of

647 acceleration and deceleration movements, by including changes of direction into the protocol elicited

648 greater movement (GPS Playerload ${ }^{\mathrm{TM}}$ ), physiological (HR), metabolic (lactate), subjective central and

649 peripheral (differential RPE) loading compared with straight line sprint running. Therefore, the

650 comparison of COD versus ST sprint exercise represented a suitable model to investigate the time-

651 course of the DNA methylome and comparative transcriptome following increased loading exercise in

652 human skeletal muscle using physiologically relevant movements predominant within many

653 sporting/exercise contexts and health prescription settings. Overall, we identified that both sprint

654 exercise conditions evoked extensive alterations in the methylome immediately post and $24 \mathrm{hrs}$ after

655 exercise, particularly in MAPK, AMPK and axon guidance pathways. COD exercise evoked a greater

656 hypomethylation response across the genome particularly enriched in: Protein binding, MAPK, AMPK,

657 insulin and axon guidance pathways, specifically immediately post exercise, compared with ST

658 exercise. Further, comparative transcriptome analysis with recent sprint running transcriptomes

659 identified considerable $49 \%$ overlap of genes altered at the expression level, and that were also

660 differentially methylated after COD exercise. In particular, after differential methylated region analysis

661 of genes altered across the methylome, we identified that vascular endothelial growth factor A

662 (VEGFA) and downstream nuclear transcription factor, NR4A1, possessed hypomethylation within

663 their promoter regions. VEGFA and NR4A1 was also significantly upregulated in both sprint

664 transcriptomes and recent comprehensive meta-analysis of 66 published exercise transcriptomes ${ }^{14}$.

665 Furthermore, within these published meta-analyses, VEGFA was highest ranked gene positively

666 correlated with well-established metabolic regulators; PCG1-alpha and NR4A3 expression in human

667 skeletal muscle after exercise ${ }^{14}$. In a re-analysis of the meta-analysis transcriptome to correspond 
668 with our study design (acute exercise immediately up to $24 \mathrm{hrs}$ post in healthy skeletal muscle), we 669 also identified significantly positively correlations of VEGFA with PCG1-alpha and NR4A3 gene 670 expression. We also confirmed increased gene expression of VEGFA at $24 \mathrm{hrs}$, PGC1- $\alpha$ and NR4A3 at $6713 \mathrm{hrs}$ post in COD exercise vs. ST exercise in the present study. In summary, we demonstrate that increased physiological load via change of direction sprint exercise in human skeletal muscle evokes considerable epigenetic modifications that are associated with changes in gene expression in genes responsible for adaptation to exercise. Further, incorporating changes in direction into exercise regimes overtime may ultimately help improve performance within a sporting context or health outcomes of populations with dysfunction in these gene pathways (MAPK, insulin signalling).

678 Acute COD sprint exercise evoked enriched hypomethylation in pathways such as insulin resistance 679 and insulin signalling (specifically immediately post exercise) that have been demonstrated to be 680 hypomethylated in skeletal muscle of people with type-II diabetes following 6 months of aerobic 681 exercise training ( 3 days/wk), 10 and in people with type-Il diabetes that are non-responders to 682 exercise ${ }^{26}$. This supports the notion that this type of change of direction sprint exercise maybe 683 beneficial for those with metabolic disease, providing this type of higher intensity exercise is tolerable 684 for these individuals. Others have suggested that it is well tolerated and also extremely practical, given 685 a reduced time-burden of the exercise regime ${ }^{27}$. Furthermore, aging is associated with 686 hypermethylation in skeletal muscle tissue ${ }^{25,28}$ and muscle stem cells ${ }^{25}$. With increased physical 687 activity and resistance exercise demonstrated to somewhat reverse the hypermethylated signature 688 observed with age ${ }^{25,29}$. Therefore, as COD exercise evokes greater hypomethylation than ST running, 689 change of direction exercise may also have a beneficial epigenomic impact in reversing the 690 hypermethylation observed in skeletal muscle tissue of aged individuals. Indeed, re-interrogation of 691 the methylome in aged skeletal muscle tissues, reveals one of the same VEGFA CpG sites (cg04629501)

692 is hypermethylated with aging ${ }^{25}$ as compared with the hypomethylated response seen with exercise 693 in the present study. Something, that requires future investigation with COD exercise in elderly 694 populations.

696 Despite COD exercise evoking a larger hypomethylation response versus straight line running. One of 697 the surprising findings of the present study was the temporal exercise alone methylome response 698 (regardless of experimental trial) demonstrated that there was a hypermethylation response 699 immediately post exercise (30 minutes), yet after $24 \mathrm{~h}$, a large majority of the same DMPs were 700 hypomethylated, suggesting a switch in the methylation status of these DMPs. Future work could 701 perhaps investigate changes in DNA methyltransferase activity at the protein level, such as DNMT3A 
and DNMT3B to investigate whether this 'transient methylation' phenomena is occurring. However,

703 it is worth mentioning that the effect of the different trials is not included in the exercise alone analysis

704 (i.e. a main effect for 'time'), that highlighted this response. Therefore, the significant differences between the trials in terms of movement patterns, physiological and metabolic loads may contribute to this finding, and therefore warrants further investigation.

707

At the candidate gene level, in the present study we identified that Nuclear Receptor Subfamily 4 Group A Member 1 (NR4A1), also known as nerve growth factor IB (NGFIB) or Nur77, was hypomethylated in its promoter in COD vs. ST exercise trials. NR4A1 is also associated with GO 'protein binding' and KEGG pathway 'MAPK signalling' identified to have enriched hypomethylation at the pathway level in COD vs. ST trials. NR4A1 is member of the steroid-thyroid hormone-retinoid receptor superfamily, where the encoded protein acts as a nuclear transcription factor. NR4A1 has been shown

714 to be increased in response to various signalling events including; cAMP, growth factors, mechanical 715 stress, cAMP, calcium, and cytokines ${ }^{30}$. NR4A1 is the most abundant nuclear receptor in skeletal 716 muscle as compared with NR4A2 and 3, and its expression is higher in fast-twitch muscle ${ }^{31}$. NR4A1 717 abundance increases with beta-adrenergic stimulation and after exercise ${ }^{31,32,33,34,35}$. NR4A1 is 718 associated with glucose uptake, glycolysis and glycogenolysis and ${ }^{31}$ its global knock-out associated 719 with an increased predisposition to obesity, insulin resistance and reduced muscle mass ${ }^{36,} 37$. 720 Overexpression of NR4A1 in skeletal muscle increases oxidative metabolism ${ }^{38}$. Indeed, NR4A1 is also significantly upregulated ( $0.94 \log$ fold) in exercise-transcriptome meta-analyses ${ }^{14}$ and within the top ten genes upregulated in the sprint transcriptome ${ }^{13}$. NR4A1 expression was also ranked $2^{\text {nd }}$ highest in its significant positive correlation with its other family member NR4A3 $(r=0.72)$ in a meta-analysis of exercise transcriptome data sets (metamex.eu ${ }^{14}$ ). Indeed, NR4A3 was the most significantly altered gene across all meta-analyses data sets and has been confirmed to be responsive to exercise stimuli in-vitro ${ }^{14}$. In the present study we also confirmed a robust 165 -fold increase in NR4A3 in COD vs. ST trail at the gene expression level. Finally, NR4A1 methylation has also been linked to epigenetic memory of skeletal muscle in the offspring of high-fat fed mothers and that this memory could be reversed if the offspring undertook exercise ${ }^{39}$. Given that hypomethylation can be retained after acute resistance exercise and training ${ }^{2}$, and given the association of NR4A1 with an epigenetic memory of nutrient stress suggests that the role of NR4A1 in an epigenetic memory of aerobic exercise in adult human skeletal muscle requires future investigation.

733 We demonstrate that Vascular Endothelial Growth Factor (VEGF), a potent angiogenic factor originally 734 described in vascular endothelial cells (also expressed in skeletal muscle), has enriched 735 hypomethylation in its promoter close to its transcription start site in change of direction versus 
straight line sprint exercise. VEGF is involved in training-induced capillary growth $40,41,42$ and is increased after acute exercise $43,44,45$ with protein levels elevated during the first 4 weeks of exercise training ${ }^{46,47}$. However, levels are lower in muscle of elderly individuals, but enhanced with training ${ }^{48,}$ 49. In the present study we also confirmed a larger increase in VEGFA gene expression at $24 \mathrm{hrs}$ in COD vs. ST trials. VEGFA was also ranked top $\left(1^{\text {st }}\right)$ most significantly correlated gene at the expression level with the canonical exercise/metabolic regulators PGC1- $\alpha(r=0.84)$ and NR4A3 $(r=0.74)$ that was the most significantly upregulated across all modes of exercise in Pillion et al., (2020) across 66 published exercise transcriptome data sets. Re-analysis of the meta-analysis transcriptome to match our study design (acute exercise immediately up to $24 \mathrm{hrs}$ post in healthy skeletal muscle), we also identified significantly positively correlations of VEGFA with PCG1-alpha and NR4A3 gene expression. We also demonstrate an increase, of a very large magnitude in PGC1- $\alpha$ (11.6 vs. 3.8-fold), as well as in NR4A3 (165.2 vs 6.8-fold) in COD vs. ST trials in the present study. VEGF mediates the upregulation of NR4A1 via activation of the PKD/HDAC7/MEF2 pathway ${ }^{50,51}$ and addition of VEGF to endothelial cells increases NR4A1, 30-fold ${ }^{52}$. Therefore, to the best of our knowledge, this is the first study to demonstrate that both VEGFA and NR4A1 are epigenetically modified (hypomethylated) and associated with an increase in gene expression after acute exercise. This hypomethylation effect is enhanced in VEGF specifically after COD exercise compared with ST exercise. Our data raises the possibility that repeated COD exercise may improve VEGFs regulation of capillary formation in response to exercise. Therefore, future studies should undertake training with COD versus ST exercise in order to confirm whether the enhanced epigenetic change in VEGF leads to improved capillary formation.

It is important to note while there was a smaller cohort used in the present study for the discovery of differentially methylated sites, the significant overlap of this methylome analysis with recent sprint transcriptome datasets and across the transcriptome meta-analyses across 66 published studies, as well the identification of an inverse relationship in methylation and gene expression, significantly strengthens the findings and the potential applicability of the results to larger cohorts.

\section{Conclusion}

We provide evidence that change of direction sprint exercise preferentially hypomethylates the skeletal muscle methylome as compared with straight line sprint exercise. Specifically, we found hypomethylation and increased gene expression of metabolic and angiogenic genes and pathways. The implication of this data suggests that introducing change of direction into high intensity running protocols could serve as an important modulator of a favourable epigenomic and transcriptomic 
landscape in response to exercise in athletes and trigger greater skeletal muscle remodelling through enhanced gene expression.

773

\section{Acknowledgments and funding}

Mohd Firdaus Maasar received a funded PhD scholarship via the Malaysian government agency: Majlis Amanah Rakyat (MARA) via Barry Drust, Adam P. Sharples and Andrew Hulton. This work was supported by the UK's Engineering and Physical Sciences Research Council (EPSRC) and the UK Medical Research Council's (MRC) centre for doctoral training, via a studentship awarded Piotr Gorski in the group of Adam P. Sharples (PI). These data were also supported by a North Staffordshire Medical Institute (NMSI), the Society for Endocrinology and Glaxosmithkline grants awarded to Adam P. Sharples (PI). Funds from Liverpool John Moores University (UK) The Norwegian School of Sport Sciences, Oslo (Norway) and The University of Birmingham (UK) supported the PhD work by Mohd and Barry Drust. Juleen R Zierath was supported from the Swedish Research Council for Sport Science (P2018-0097) and the Swedish Research Council (Vetenskapsrådet) (2015-00165). Nicolas J. Pillon was supported by an Individual Fellowship from the Marie Skłodowska-Curie Actions (European Commission, 704978).

\section{Declaration}

All authors declare no conflicts of interest.

791

\section{References}

1. Turner DC, Seaborne RA, Sharples AP. Comparative Transcriptome and Methylome Analysis in Human Skeletal Muscle Anabolism, Hypertrophy and Epigenetic Memory. Scientific reports $\mathbf{9}$,

2. Seaborne RA, et al. Human Skeletal Muscle Possesses an Epigenetic Memory of Hypertrophy. Scientific Reports (Nature) 8, 1898 (2018).

3. Seaborne RA, et al. Methylome of human skeletal muscle after acute \& chronic resistance

4. Seaborne RA, et al. UBR5 is a novel E3 ubiquitin ligase involved in skeletal muscle hypertrophy 
810 6. Lister R, et al. Human DNA methylomes at base resolution show widespread epigenomic differences. Nature 462, 315-322 (2009).

812

813 7. Ziller MJ, et al. Charting a dynamic DNA methylation landscape of the human genome. Nature 500, 477-481 (2013).

815

816

8. Bogdanovic O, Veenstra GJ. DNA methylation and methyl-CpG binding proteins: developmental requirements and function. Chromosoma 118, 549-565 (2009).

818

819

9. Lindholm ME, et al. An integrative analysis reveals coordinated reprogramming of the epigenome and the transcriptome in human skeletal muscle after training. Epigenetics : official journal of the DNA Methylation Society 9, 1557-1569 (2014).

10. Nitert MD, et al. Impact of an exercise intervention on DNA methylation in skeletal muscle

11. Rowlands DS, et al. Multi-omic integrated networks connect DNA methylation and miRNA with skeletal muscle plasticity to chronic exercise in Type 2 diabetic obesity. Physiological Genomics 46, 747-765 (2014).

12. Barres R, et al. Acute exercise remodels promoter methylation in human skeletal muscle. Cell

13. Rundqvist HC, Montelius A, Osterlund T, Norman B, Esbjornsson M, Jansson E. Acute sprint

14. Pillon NJ, et al. Transcriptomic profiling of skeletal muscle adaptations to exercise and

15. Nicolella DP, Torres-Ronda L, Saylor KJ, Schelling X. Validity and reliability of an accelerometer-

16. Weston M, Siegler J, Bahnert A, McBrien J, Lovell R. The application of differential ratings of

17. Maksimovic J, Phipson B, Oshlack A. A cross-package Bioconductor workflow for analysing

18. Pidsley R, et al. Critical evaluation of the Illumina MethylationEPIC BeadChip microarray for whole-genome DNA methylation profiling. Genome biology 17, 208 (2016). 
20. Du $\mathrm{P}$, et al. Comparison of Beta-value and $\mathrm{M}$-value methods for quantifying methylation levels by microarray analysis. BMC Bioinformatics 11, 587 (2010).

21. Kanehisa M, Goto S. KEGG: kyoto encyclopedia of genes and genomes. Nucleic Acids Res 28, 27-30 (2000).

22. Kanehisa M, Furumichi M, Tanabe $M$, Sato $Y$, Morishima K. KEGG: new perspectives on genomes, pathways, diseases and drugs. Nucleic Acids Res 45, D353-d361 (2017).

23. Kanehisa M, Sato Y, Kawashima M, Furumichi M, Tanabe M. KEGG as a reference resource for gene and protein annotation. Nucleic Acids Res 44, D457-462 (2016).

24. Schmittgen TD, Livak KJ. Analyzing real-time PCR data by the comparative C(T) method. Nature protocols 3, 1101-1108 (2008).

25. Turner DC, et al. DNA methylation across the genome in aged human skeletal muscle tissue and muscle-derived cells: The role of HOX genes and physical activity. Scientific Reports (Nature) Accepted in Press https://doi.org/10.1038/s41598-020-72730-z, (2020).

26. Stephens NA, et al. Exercise Response Variations in Skeletal Muscle PCr Recovery Rate and Insulin Sensitivity Relate to Muscle Epigenomic Profiles in Individuals With Type 2 Diabetes. Diabetes Care 41, 2245-2254 (2018).

27. Liu J-X, Zhu L, Li P-J, Li N, Xu Y-B. Effectiveness of high-intensity interval training on glycemic control and cardiorespiratory fitness in patients with type 2 diabetes: a systematic review and meta-analysis. Aging clinical and experimental research 31, 575-593 (2019).

28. Zykovich A, et al. Genome-wide DNA methylation changes with age in disease-free human skeletal muscle. Aging cell 13, 360-366 (2014).

29. Blocquiaux S, et al. Recurrent training rejuvenates and enhances transcriptome and methylome responses in young and older human muscle. bioRxiv, DOI: 10.1101/2020.1106.1130.179465 (2020).

30. Maxwell MA, Muscat GE. The NR4A subgroup: immediate early response genes with pleiotropic physiological roles. Nucl Recept Signal 4, e002 (2006).

31. Chao LC, Zhang Z, Pei L, Saito T, Tontonoz P, Pilch PF. Nur77 coordinately regulates expression of genes linked to glucose metabolism in skeletal muscle. Mol Endocrinol 21, 2152-2163 (2007).

32. Mahoney DJ, Parise G, Melov S, Safdar A, Tarnopolsky MA. Analysis of global mRNA expression in human skeletal muscle during recovery from endurance exercise. Faseb j 19, 1498-1500 (2005). 
900

901

902

903

904

905

906

907

908

909

910

911

912

913

914

915

916

917

918

919

920

921

922

923

924

925

926

927

928

929

930

931

932

933

934

935

936

937

938

939

940

941

942

33. Myers SA, Eriksson N, Burow R, Wang SC, Muscat GE. Beta-adrenergic signaling regulates NR4A nuclear receptor and metabolic gene expression in multiple tissues. Mol Cell Endocrinol 309, 101-108 (2009).

34. Catoire $\mathrm{M}$, et al. Pronounced effects of acute endurance exercise on gene expression in resting and exercising human skeletal muscle. PLoS ONE 7, e51066 (2012).

35. WuX, et al. The effect of insulin on expression of genes and biochemical pathways in human skeletal muscle. Endocrine 31, 5-17 (2007).

36. Chao LC, et al. Insulin resistance and altered systemic glucose metabolism in mice lacking Nur77. Diabetes 58, 2788-2796 (2009).

37. Tontonoz $\mathrm{P}$, et al. The orphan nuclear receptor Nur77 is a determinant of myofiber size and muscle mass in mice. Mol Cell Biol 35, 1125-1138 (2015).

38. Chao LC, et al. Skeletal muscle Nur77 expression enhances oxidative metabolism and substrate utilization. J Lipid Res 53, 2610-2619 (2012).

39. Kasch J, et al. Insulin sensitivity linked skeletal muscle Nr4a1 DNA methylation is programmed by the maternal diet and modulated by voluntary exercise in mice. The Journal of nutritional biochemistry 57, 86-92 (2018).

40. Lloyd PG, Prior BM, Li H, Yang HT, Terjung RL. VEGF receptor antagonism blocks arteriogenesis, but only partially inhibits angiogenesis, in skeletal muscle of exercise-trained rats. Am J Physiol Heart Circ Physiol 288, H759-768 (2005).

41. Delavar H, Nogueira L, Wagner PD, Hogan MC, Metzger D, Breen EC. Skeletal myofiber VEGF is essential for the exercise training response in adult mice. Am J Physiol Regul Integr Comp Physiol 306, R586-595 (2014).

42. Olfert IM, Howlett RA, Wagner PD, Breen EC. Myocyte vascular endothelial growth factor is required for exercise-induced skeletal muscle angiogenesis. Am J Physiol Regul Integr Comp Physiol 299, R1059-1067 (2010).

43. Breen EC, Johnson EC, Wagner H, Tseng HM, Sung LA, Wagner PD. Angiogenic growth factor mRNA responses in muscle to a single bout of exercise. J Appl Physiol (1985) 81, 355-361 (1996).

44. Gustafsson T, Sundberg CJ. Expression of angiogenic growth factors in human skeletal muscle in response to a singular bout of exercise. Am J Physiol Heart Circ Physiol 279, H3144-3145 (2000). 
45. Richardson RS, Wagner H, Mudaliar SR, Henry R, Noyszewski EA, Wagner PD. Human VEGF gene expression in skeletal muscle: effect of acute normoxic and hypoxic exercise. Am J Physiol 277, H2247-2252 (1999).

46. Gustafsson T, et al. Increased expression of vascular endothelial growth factor in human skeletal muscle in response to short-term one-legged exercise training. Pflugers Arch 444, 752-759 (2002).

47. Hoier B, et al. Pro- and anti-angiogenic factors in human skeletal muscle in response to acute exercise and training. J Physiol 590, 595-606 (2012).

48. Croley AN, et al. Lower capillarization, VEGF protein, and VEGF mRNA response to acute exercise in the vastus lateralis muscle of aged vs. young women. J Appl Physiol (1985) 99, 1872-1879 (2005).

49. Gliemann L, et al. Resveratrol modulates the angiogenic response to exercise training in skeletal muscles of aged men. Am J Physiol Heart Circ Physiol 307, H1111-1119 (2014).

50. Wang S, Li X, Parra M, Verdin E, Bassel-Duby R, Olson EN. Control of endothelial cell proliferation and migration by VEGF signaling to histone deacetylase 7. Proc Natl Acad Sci U S A 105, 7738-7743 (2008).

51. $\mathrm{Ha} \mathrm{CH}$, Jhun BS, Kao HY, Jin ZG. VEGF stimulates HDAC7 phosphorylation and cytoplasmic accumulation modulating matrix metalloproteinase expression and angiogenesis. Arteriosclerosis, thrombosis, and vascular biology 28, 1782-1788 (2008).

52. Ismail $\mathrm{H}$, et al. Angiopoietin-1 and vascular endothelial growth factor regulation of leukocyte adhesion to endothelial cells: role of nuclear receptor-77. Arteriosclerosis, thrombosis, and vascular biology 32, 1707-1716 (2012). 
$1 a$

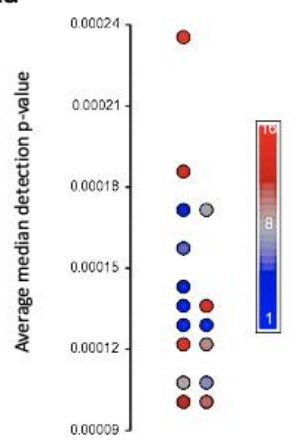

1c

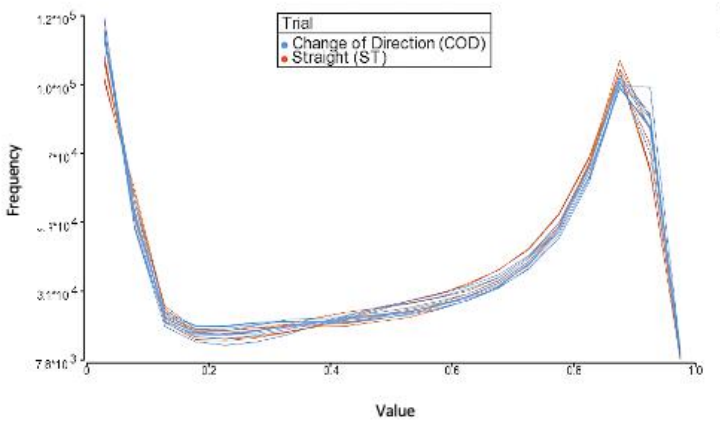

$1 b$

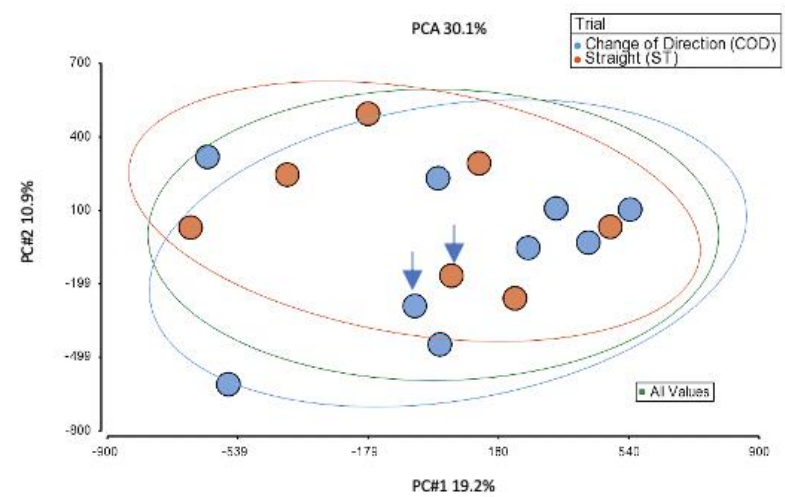

$1 d$

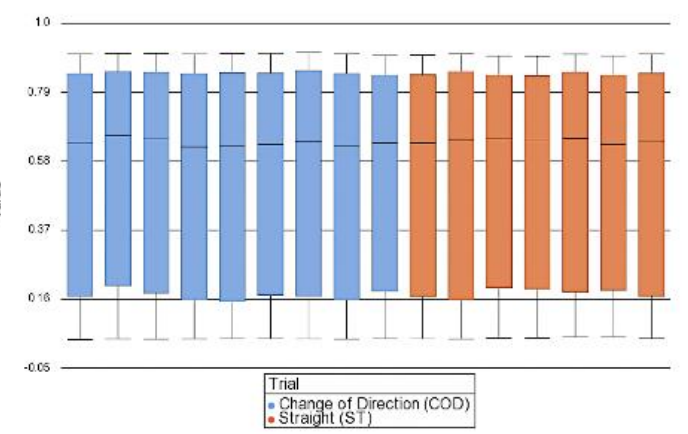

Suppl. Figure 1. 